\title{
Determination of Erucic acid content in traditional and commercial mustard oils of Bangladesh by Gas- Liquid Chromatography
}

\author{
Md. Tanvir Sarwar ${ }^{1}$, Md. Hafizur Rahman', Md. Salim Raza ${ }^{1}$, Shakh M. A. Rouf ${ }^{1}$, \\ Md. Nazibur Rahman ${ }^{2}$ \\ ${ }^{1}$ Department of Applied Nutrition and Food Technology, Islamic University, Kushtia-7003, Bangladesh \\ ${ }^{2}$ Department of Biochemistry and Molecular Biology, Jahangirnagar University, Savar, Dhaka-1342, Bangladesh \\ Email address: \\ tanvirsarwariu@gmail.com (T. Sarwar)
}

To cite this article:

Md. Tanvir Sarwar, Md. Hafizur Rahman, Md. Salim Raza, Shakh M. A. Rouf, Md. Nazibur Rahman. Determination of Erucic Acid Content in Traditional and Commercial Mustard Oils of Bangladesh by Gas- Liquid Chromatography. Advances in Biochemistry. Vol. 2, No. 1, 2014, pp. 9-13. doi: 10.11648/j.ab.20140201.12

\begin{abstract}
The modern trend of consuming mustard oil in raw, fried and cooked forms is increasing day by day in the general people irrespective of economic status in Bangladesh. But unfortunately they are not aware of the adverse effects of this oil. Because, mustard oils have been evidenced to have high level of erucic acid and erucic acid has been evidenced to be a causative agent for cardiovascular diseases. It is therefore very important to know the erucic acid level both in traditional and commercially available mustard oils in Bangladesh. The aim of the study was to investigate the fatty acid profile of the commercial mustard oil (Industrially manufactured) and ghani (traditional method of oil processing) mustard oil. After preparation of the purified fatty acid methyl esters of the above oils, Gas-Liquid Chromatographic (GLC) analysis was carried out. Results showed that the percentage of erucic acid (22:1) in the commercial and ghani mustard oils were $41.80 \%$ and $51.98 \%$ respectively. In context to percentage of erucic acid, the commercial mustard oil seemed better compared to ghani. The reasons behind this discrepancy still remains unclear but the possibility of mixed oil effects in the commercial mustard oil could be investigated in the near future. The author also established a method to partially eliminate erucic acid from mustard oil. After partially eliminate erucic acid from commercial mustard oil erucic acid reduces from $41.80 \%$ to $20.14 \%$.
\end{abstract}

Keywords: Mustard Oil, Fatty Acid, Erucic Acid

\section{Introduction}

Edible vegetable oils are the chief source of nutritionally required fatty acids in human diet. Mustard oil, soybean oil, sunflower oil and groundnut oil are among the edible vegetable oils mostly consumed in Bangladesh. However, none of these oils alone provide many of the lipid soluble nutrients as per the recommendation of health agencies. Mustard oil is extracted from the seeds of mustard plant (Brassica campestrisL.), belonging to the cruciferous family of plants. It is originally from the Mediterranean regions, but it grows easily in many parts of the world. It has a characteristically pungent flavor and aroma. Though this oil is nutty tasting it is good for heart and also has many other benefits. Mustard oil contains a high amount of selenium and magnesium, which gives it anti-inflammatory properties. It also helps stimulating sweat glands and helps lowering body temperature. In traditional medicines, it is used to relieve the pain associated with arthritis, muscle sprains and strains. Seed paste applied on wounds whereas paste of leaf said to heal cattle wounds ${ }^{1}$. Mustard oil makes up about $24-40 \%$ of the mustard seeds, which is characterized by the presence of higher level of erucic acid and it has the lowest saturated fatty acids content among all the edible vegetable oils. Mustard oil contains fatty acids like erucic, oleic, linoleic and $\alpha$-linolenic acid. Rapeseed-mustard consists of saturated fatty acid such as palmitic (C16:0), stearic (C18:0) and monounsaturated fatty acids such as oleic (18:1) eicosenoic (C 20:1) and erucic acid (C 22:1) and polyunsaturated fatty acids such as linoleic (C 
18:2) and linolenic acid (C 18:3), known as essential fatty acids. Of the total fatty acids, erucic acid (C 22:1) is predominant in Indian cultivars (about 50 per cent). The presence of high erucic acid in oil is considered anti-nutritional, as it has been reported to cause lipidosis in children and myocardial fibrosis in monkeys ${ }^{3}$. Erucic acid (EA) is the common name for cis-13-docosenoic acid, a typical example of a very-longchain mono-unsaturated fatty acid (VLCMFA). EA has 22 carbon atoms in its straight-chain molecule and one double bond between $\mathrm{C} 13$ and $\mathrm{C} 14$.The major source of EA is the high-erucic-acid (HEA) seed oils of the Crucifereae family, which includes rapeseed, mustard, crambe, and wallflower, all containing about $45 \%$ to $60 \% \mathrm{EA}^{4}$. However, higher levels of erucic acid are unsuitable for human consumption as food purposes $^{5}$. Higher consumption of erucic acid may increase the concentration of adrenal cholesterol causing fibrotic changes in myocardium, liver weight and cholesterol ${ }^{6,7}$. High levels of erucic acid is not suitable for human food in European Union and other developed countries since erucic acid showed serious pathological changes in the heart and skeletal in animals ${ }^{8}$. In spite of the concern about its safety for human consumption, EA is a very important raw material in the oleo chemical industry. Researchers have found that EA and its derivatives possess varieties of superior properties in slipping, softening, antifoaming, emulsifying, and corrosion inhibiting. All these properties offer EA and its derivatives wide applications in the production of pharmaceuticals, soaps, detergents, cosmetics, plastics, lubricants, rubbers, coatings, etc ${ }^{9,}{ }^{10}$. Rapeseed is the third leading source of edible oil in the world. Rapeseed oil (RSO) is the most useful of all cooking oils and it contains a significant amount of n-3 and n- 6 fatty acids. RSO contains mostly of the fatty acid such as oleic, linoleic, linolenic, palmitic and strearic acid ${ }^{11,12}$. The oil content usually makes upabout $40-60 \%$ of rapeseed. RSO consists $95 \%$ oftriacylglycerols (TAG) and 5\% non-triacylglycerols, known as minor components like free fatty acids, mono- and diacylglycerols, phospholipids, tocopherols, tocotrienols, flavonoids, other phenolic compounds, pigments (chlorophylls), sterols etc ${ }^{13}$.

\section{Materials \& Methods}

\subsection{Oils and Chemicals}

Mustard oil used in the present was collected from two sources. First one was commercial mustard oil, collected from open market of Jhenaidah district of Bangladesh. The second type of oil was ghani mustard oil (traditional mustard oil) and collected from Sheikhpara ghani mill, Kushtia, Bangladesh.

The Sodium chloride acid obtained from the Fisher Scientific Company, Fairlawn, New Jersey, USA. Nitrogen gas and Glacial acetic acid from Bangladesh Oxygen Ltd, Dhaka, Bangladesh. Standard fatty acid methyl esters from SIGMA Chemical Co, St. Loius, USA.

\subsection{Preparation of Anhydrous Methanolic HCL (5\% w/w)}

Traces of water were removed from methanol by allowing it to stand overnight in contact with fused anhydrous coarse powdered calcium chloride and then either filtering or centrifuging the suspension. The dry methanol thus obtained was stored in a tightly stopper brown bottle. Methanol $\mathrm{HCl}$ mixture was prepared in the following manner. Hydrochloric acid gas also dry was generated from $\mathrm{NaCl}$ contained in a quick fit round bottom flask, with concentrated $\mathrm{H}_{2} \mathrm{So}_{4}$ added to the salt drop by drop from a burette. The gas produced was passed through another round bottom flask filled with the fused $\mathrm{CaCl}_{2}$ powder.

The gas was introduced into a known amount of dry methanol contained in a reagent bottle. The gas was passed until it was at least $5 \%(\mathrm{w} / \mathrm{w})$ in solvent (methanol). Usually a $7 \% \mathrm{HCl}$ in methanol mixture was prepared. Traces of $\mathrm{CaCl}_{2}$ powder were found to sediment on standing and were removed by filtration. This methanolic $\mathrm{HCl}$ reagent was stored in a tightly stopper bottle.

\subsection{Methylation of Fatty Acid}

Total lipid (400-600 mg) was taken in a ground joint flask and saponified with $15-30 \mathrm{~mL} 2 \mathrm{M} \mathrm{KOH}$ (ethanolic) in water bath at $70^{\circ} \mathrm{C}$ for 1 hour by joining with a condenser. After cooling, the solution was diluted with equal volume of distilled water and acidified with concentrated $\mathrm{HCl}$ to $\mathrm{P}^{\mathrm{H}}<2$ as ascertained with a $\mathrm{P}^{\mathrm{H}}$ meter. The liberated fatty acids (a mixture) were extracted with $30-60 \mathrm{~mL}$ of diethyl ether. Small amount of water was also extracted along with free fatty acids. This undesired water was removed by adding anhydrous sodium sulphate. The ether extract devoid of water was collected in another joint flask. The extract was then evaporated to dryness under $\mathrm{N}_{2}$. Dry methanolic $\mathrm{HCl}$ (25-50 $\mathrm{mL}$ ) prepared as above, was added into the fask containing the fatty acid mixture and the content was heated at $85^{\circ} \mathrm{C}$ under reflux for 2 hours. After cooling, the fatty acids methyl esters (FAME) were extracted three times with equal volume of petroleum spirit (bp40-60 $)$. All extracts were combined and evaporated to a small volume under $\mathrm{N}_{2}$.

\subsection{Isolation of Erucic Acid from Mustard Oil}

\subsubsection{Saponification}

Two 600 -g batches of bleached oil were each divided into three 200-g samples and saponified under re- flux. The procedure was a modification of AOCS method Cd 3-25 (16) using water in place of ethanol and $\mathrm{NaOH}$ in place of $\mathrm{KOH}$. On the basis of the saponification value, $350 \mathrm{~mL}$ of aqueous $2 \mathrm{M} \mathrm{NaOH}$ solution, providing a $13 \%$ excess, was added with stirring to $200 \mathrm{~g}$ warm $\left(50^{\circ} \mathrm{C}\right)$ bleached oil in a 4-L flask. The mixture was boiled at $108-112{ }^{\circ} \mathrm{C}$ with reflux for $6 \mathrm{~h}$ until the sample became a homogeneous, milky liquid giving a heavy, white, soapy upper layer upon cooling. The contents were cooled for $1 \mathrm{~h}$ at room temperature $\left(23^{\circ} \mathrm{C}\right)$ and $4 \mathrm{~h}$ at $6^{\circ} \mathrm{C}$. The alkali liquor was drained from the cake of soap stock. 


\subsubsection{Acidification}

The soap stock was slurried with one-half volume of $30 \%$ (w/w) $\mathrm{HCl}$, then additional acid was added and the mixture was brought to a boil. The acid solution was added slowly and with constant agitation until the soaps were dissolved and $\mathrm{pH} 1$ was achieved. This yielded a brownish- yellow mixture of free fatty acid (FFA) and acid liquor which was cooled for $1 \mathrm{~h}$ at $23{ }^{\circ} \mathrm{C}$ and $4 \mathrm{~h}$ at $68 \mathrm{C}$ to produce two well-separated phases without emulsion. The upper FFA layer was removed and gently washed with $300 \mathrm{~mL}$ distilled water.

\subsubsection{Isolation of Erucic Acid}

The FFA was dissolved in $95 \%$ ethanol ( $6 \mathrm{~mL} / \mathrm{g}$ of FFA) at $38-42{ }^{\circ} \mathrm{C}$ on a steam bath, and cooled to the crystallization temperature $\left(-14{ }^{\circ} \mathrm{C}\right)$ in a walk-in freezer. The mixture was allowed to crystallize overnight. The crystals were recovered from the mixture on Whatman No. 1 filter paper, washed twice with $100 \mathrm{~mL}$ of cold $95 \%$ ethanol, then held over vacuum on an unchilled Buchner funnel until the last traces of alcohol were removed. The alcoholic filtrate was held at $-14 \circ \mathrm{C}$ for an additional $1 \mathrm{~d}$, producing a second crop of crystals which were recovered, rinsed, and dried as described above.

The first and second crops were combined to form the first crystallization product. A second crystallization was carried out by dissolving the first crystallization product in $95 \%$ ethanol ( $6 \mathrm{~mL} / \mathrm{g}$ of FFA), cooling the solution to $-14 \circ \mathrm{C}$, and collecting the crystals as described for the first crystallization, except that a second crop of crystals was not collected.

\subsection{Purification of Fatty Acid Methyl Esters (FAME)}

\subsubsection{Preparation of TLC Plate}

A slurry of silica gel $\mathrm{G}$ for thin layer chromatography is made with water $(2 \mathrm{~mL}$ water per gm silica gel $\mathrm{G})$ in a beaker (500mL capacity) and spread on $2 \mathrm{~mm}$ thick glass plates $20 \times 20 \mathrm{~cm}$ by a TLC spreader. The silica gel coating is 250 $\mu \mathrm{m}$. The slurry thus spread is kept on the platform for about 10 minutes, transfer to the metal racks and dried in an oven at $110^{\circ} \mathrm{C}$ for about an hour. The plates are now ready for use.

\subsubsection{Thin Layer Chromatographic (TLC) Procedure}

Standard fatty acids preparation $(\sim 3-5 \mathrm{~mL})$ is now spotted on the plates with a glass capillary taking precaution so that not more than $2-3 \mu \mathrm{L}$ are spotted on the plates at a distance nearly $3 / 4$ for an inch from one edge on the plates. The gaps between two spots should be around half an inch and the spots should be as small as possible for better resolution of the fatty acids. The unknown should be spotted on the two locations. After air drying the plate is dipped in the solvents (n- hexane: Diethyl ether: glacial acetic acid 70:30:1) in the TLC jar which is pre-equilibrated with the solvent system for about an hour. The solvent rise up the silica gel (ascending chromatography) and is allowed to rise approximately anywhere between 15-18 cm (nearly one hours) at which point the plate is removed from the jar, air dried, placed in the iodine chamber for 5 minutes. The FAME band in the plate was visualized in the iodine chamber. The FAME in the sample can be identified by their $\mathrm{R}_{\mathrm{f}}$ values when compared to standard. After the yellow color vanished the band was scraped into a centrifuge tube and eluted with methanol. The tube was then centrifuged and the supernatant was transferred into a dry flask. The FAME solution was evaporated to dryness under nitrogen. A small volume of dichloromethane solution was added to re-dissolve the FAME band and a 5-10 $\mathrm{mm}$ aliquot was analyzed in Gas-liquid chromatography.

$$
\mathrm{R}_{\mathrm{f}}=\frac{\text { Distance Traveled by particularFatty Acid }}{\text { Disyance Traveled by Solvent front }}
$$

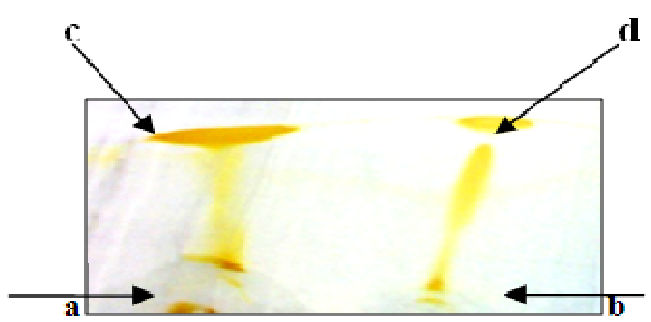

Figure1.Photograph showing methyl ester band of fatty acids in a TLC plate.

In Fig- 2, a= Left arrow (bottom): Spot of non-purified methyl esters of fatty acids, $c=$ Left arrow (top): Band of methyl esters (pure) of fatty acids, $b=$ Right arrow (bottom): Spot of standard methyl oleate, $\mathrm{d}=$ Right arrow (top): Band of standard methyl oleate.

\subsection{Gas-Liquid Chromatographic (GLC) analysis of fatty acid methyl esters}

The fatty acid methyl esters, prepared and purified as above, were analyzed by gas-liquid chromatography (GLC). A $2 \times 4 \mathrm{~mm}$ inside diameter column (Preferably glass) packed with $12-15 \%(\mathrm{w} / \mathrm{w})$ ethylene glycol succinate liquid phase coated on 100/200 mesh Gas-chrom P was used. The injector temperature was $190^{\circ} \mathrm{C}$ and the detector temperature was $260^{\circ} \mathrm{C}$. The temperature of the column was programmed initially at $170^{\circ} \mathrm{C}$ for 8 minutes, then it was allowed to rise to $200^{\circ} \mathrm{C}$ at a rate of $1^{0} \mathrm{C} / \mathrm{min}$ and the isothermal final period was 55 minutes. Thermal conductivity detectors were excellent. Nitrogen was used as a carrier gas at a flow rate of $11.4 \mathrm{~mL} / \mathrm{min}$. Hydrogen flow was $10 \%$ above nitrogen flow. Standard fatty acid methyl esters were used for the identification of the sample fatty acid peaks. The following Standard fatty acids were used, the methyl esters of $\mathrm{C}_{8: 0} \mathrm{C}_{9: 0}$ $\mathrm{C}_{10: 0} \mathrm{C}_{11: 0} \mathrm{C}_{12: 0} \mathrm{C}_{14: 0} \mathrm{C}_{16: 0} \mathrm{C}_{18: 0} \mathrm{C}_{18: 1} \mathrm{C}_{18: 2} \mathrm{C}_{18: 3} \mathrm{C}_{20: 0} \mathrm{C}_{22: 0 .}$ The peak area of each component was measured automatically by chromatograph machine. It was also measured by the actual physical measurement by the triangulation method (Yusuf et al, 1981). The total mm of all peak areas were taken as $100 \%$ and the percent population of a given fatty acid peak was calculated accordingly. The fatty acids were expressed as weight percentages of total fatty acids. 
12 Md. Tanvir Sarwar et al.: Determination of Erucic Acid Content in Traditional and Commercial Mustard Oils of Bangladesh by Gas- Liquid Chromatography

\section{Results}

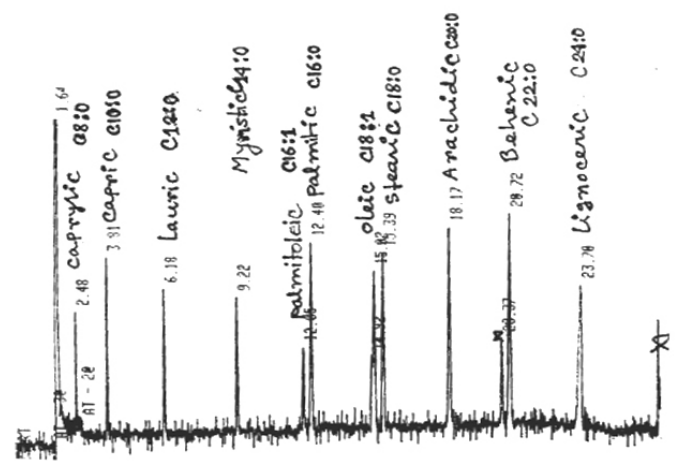

Fig 2.The GLC line graph of the standard fatty acids as- $C_{8: 10} C_{10: 0} C_{12: 0} C_{14: 0}$ $C_{16: 0} C_{18: 0} C_{18: 1} C_{20: 0} C_{22: 0} C_{24: 0}$

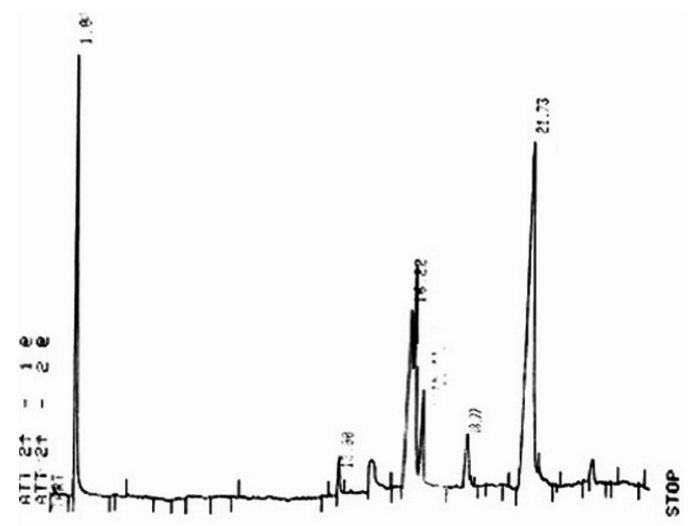

Fig3. Gas chromatogram of commercial mustard oil. Description of peaks is as follows:(13.00 Palmitic acid), (16.22 Oleic acid), (16.41 Stearic acid), (18.77 Arachidic acid), (21.73 Erucic acid)

Table1. Fatty acid composition of Commercial mustard oil measured on GLC

\begin{tabular}{ccc}
\hline Retention Time & Name of Acid & Relative \% \\
\hline 13.00 & Palmitic Acid & 1.84 \\
16.22 & Oleic Acid & 16.66 \\
16.41 & Stearic Acid & 3.43 \\
18.77 & Arachidic Acid & 2.03 \\
21.73 & Erucic Acid & 41.80 \\
\hline
\end{tabular}

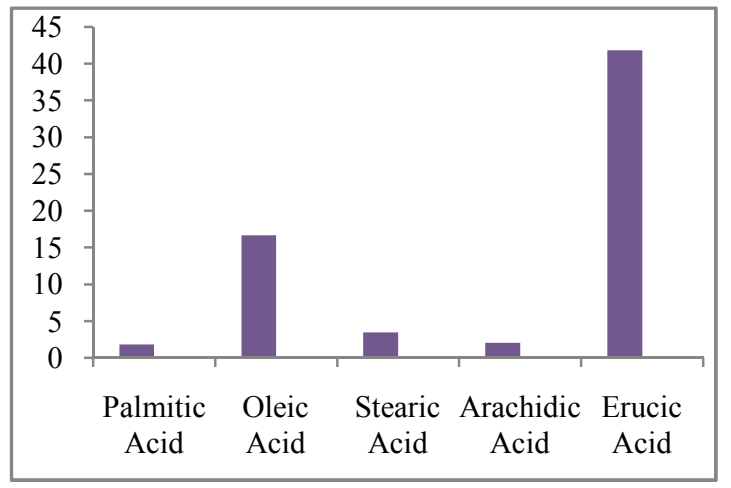

Fig 4. Fatty acid profile of commercial mustard oil .

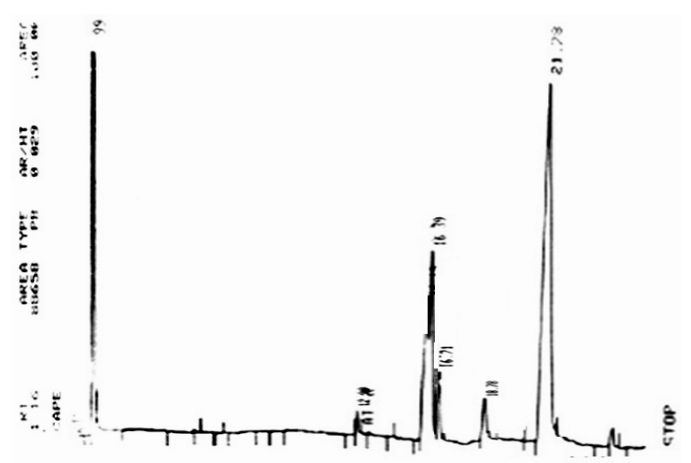

Fig 5. Gas chromatogram of ghani mustard oil. Description of peaks is as follows:(12.99 Palmitic acid), (16.39 Oleic acid), (16.71 Stearic acid), (18.78 Arachidic acid), (21.66 Erucic acid)

Table2. Fatty acid composition of ghani (traditional mustard oil) mustard oil measured on GLC

\begin{tabular}{ccc}
\hline Retention Time & Name of Acid & Relative \% \\
\hline 12.99 & Palmitic Acid & 1.99 \\
16.39 & Oleic Acid & 17.04 \\
16.71 & Stearic Acid & 3.60 \\
18.78 & Arachidic Acid & 2.46 \\
21.78 & Erucic Acid & 51.98 \\
\hline
\end{tabular}

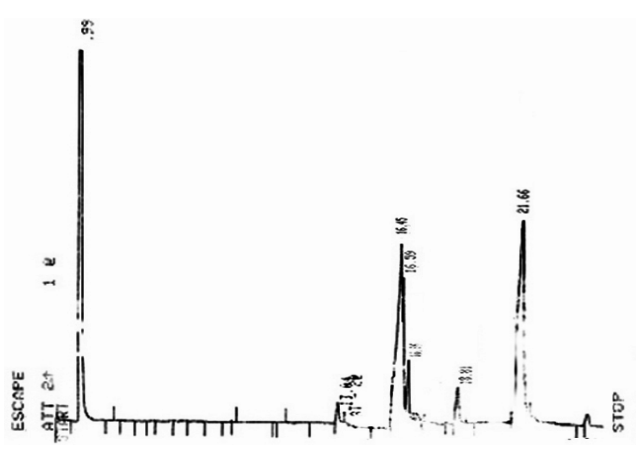

Fig 6. Gas chromatogram of erucic free commercial mustard oil. Description of peaks is as follows :(13.04 Palmitic acid), (16.45 Oleic acid), (16.86 Stearic acid), (18.81 Arachidic acid), (21.66 Erucic acid)

Table3. Fatty acid composition of erucic free commercial mustard oil measured on GLC

\begin{tabular}{ccc}
\hline Retention Time & Name of Acid & Relative \% \\
\hline 13.04 & Palmitic Acid & 1.35 \\
16.45 & Oleic Acid & 16.55 \\
16.86 & Stearic Acid & 3.29 \\
18.81 & Arachidic Acid & 2.03 \\
21.66 & Erucic Acid & 20.14 \\
\hline
\end{tabular}

\section{Discussion}

Vegetable oils in human diet constitute an important source of energy and have considerable importance in human health. Besides being the gastronomic delights and a source of energy, edible vegetable oils provide fat-soluble micronutrients and essential and nonessential fatty acids, which perform various functions. Besides the fatty acids, unsaponifiable matter is a major chunk of edible vegetable 
oils. This includes various micronutrients such as tocopherols, tocotrienols, carotene, oryzanol, squalene etc. depending on the type of the edible oil. The micronutrients in vegetable oils have various functions. Tocopherols, for example, are recognized for the antioxidant activity, and have been observed to be useful in degenerative diseases like cancer, cataract, cardiovascular disease and ageing. Tocopherols in oil actthrough several mechanisms such as inhibiting the lipid peroxidation, chain termination, singlet oxygenquenching, and radical scavenging to deactivate free radicals that are produced during the oxidation of biomolecules $^{14}$.

In the present study two types of mustard oils (commercial mustard oil and ghani oil) were analyzed by GLC to study their fatty acid profiles. The GLC analysis (Table 1) revealed that commercial mustard oil represents $1.84 \%$ palmitic acid, $16.66 \%$ oleic acid, $3.43 \%$ stearic acid, $2.03 \%$ arachidic acid and $41.80 \%$ erucic acid (22:1). On the other hand ghani (traditional method of oil processing) mustard oil represents (Table-2) 1.99\% palmitic acid, $17.04 \%$ oleic acid, $3.60 \%$ stearic acid, $2.46 \%$ arachidic acid and $51.98 \%$ erucic acid (22:1). Erucic acid possesses a very larger percentage in both the samples of mustard oil. But erucic acid percentage of commercial mustard oil is lower than the ghani mustard oil. The author here tried to partially eliminate erucic acid from commercial mustard oil. After applying the elimination process on commercial mustard oil we found (Table-3) 1.35\% palmitic acid, $16.55 \%$ oleic acid, $3.29 \%$ stearic acid, $2.03 \%$ arachidic acid and $20.14 \%$ erucic acid (22:1). Of the total fatty acids of mustard oil, erucic acid (C 22:1) is predominant in Bangladeshi cultivars (about 50 per cent). The presence of high erucic acid in oil is considered anti-nutritional, as it has been reported to cause lipidosis in children and myocardial fibrosis in monkeys ${ }^{15}$. Though the author tried to establish a laboratory method to partially eliminate harmful erucic acid from the mustard oil, there is an urgent need to make concerted efforts to eliminate total erucic acid from mustard oil.

\section{References}

[1] S.K. Sood, D. Sharma, S. Kumar, and T.N. Lakhanpal, Healing Herbs: Traditional Medications for Wounds, Sores and Bones. Pointer Publishers, Jaipur, India, 2010.

[2] USDA National Nutrient Database. Entry for mustard oil in the USDA National Nutrient Database for Standard Reference, Release 22.http://www.nal.usda.gov/fnic/foodcomp/cgi-bin/measure.
pl?MSRE $\mathrm{NO}=04583 \times y z 0400 \times y z O i 1 \% 252 \mathrm{c} \% 2520$ mustard xyzxyz.

[3] Ackman, R.G.; Eaton, C.A.; Sipos J.C.; Loew F.W. and Hancock, D. Comparison of fatty acid from high levels of erucic acid of RSO and partially hydrogenated fish oil in non-human primate species in a short term exploratory study. Nutr. Diet. 25; 170-185,1977.

[4] Sonntag, N. O. V. Erucic, behenic: Feedstocks of the $21^{\text {st }}$ century. INFORM 2(5): 449-463.1991.

[5] Kramer, J. K. G., E. R. Farnworth, B. K. Thompson, A. H. Corner and H. L. Trenholm. Reduction of myocardial necrosis in male albino rats by manipulation of dietary fatty acid levels. Lipids 17(5):372-382,1982.

[6] Aaes-Jorgensen, E. Nutritional value of rapeseed oil, in Rapeseed Cultivation, Composition, Processing and Utilization, 286 by Appelqvist, L. A. and Ohlson, R., Elsevier, Amsterdam. pp.301-353,1972.

[7] Beare-Rogers, J. L., E. A. Nera and B. M. Craig. Cardiac lipids of rats and gerbils fed oils containing $\mathrm{C} 22$ fatty acids. Lipids 7:548-552, 1972.

[8] Food Standards Australia New Zealand. Erucic acid in food: A toxicological review and risk assessment. Tech. Report Series. 21:1448-3017,2003.

[9] Carlson, K. D., and D. L. Van Dyne. Industrial uses for high erucic acid oils from crambe and rapeseed. Columbia, Mo.:University of Missouri-Columbia,1992.

[10] Erickson, D. B., and P. Bassin. Rapeseed and crambe: Alternative crops with potential industrial uses. Bulletin 656 Manhattan, Kansas: Kansas State University, Agricultural Experiment Station,1997.

[11] Gunstone, F. D., J. L. Harwood and F. B. Padley.The Lipid Handbook (2nd ed.). Chapman\& Hall, Chemical Database, London. pp.119,1994.

[12] Hui, Y. H. Bailey's Industrial Oil and FatProducts. Edible oil and fat products: Generalapplications (5th ed.). 1, John Wiley \& SonsInc. NY. pp 25,1996.

[13] Shahidi, F. and V. K. S. Shukla. .Nontriacylglycerol constituents of fats andoils. Internat. News Fats, Oils RelatedMaterials 7:1227-1231,1996.

[14] A.A. Qureshi, N. Qureshi, J.J. Wright, Z. Shen, G. Kramer, A. Gapor, Y.H.Chong, G. DeWitt, A.Ong, and D.M. Peterson, Am. J. Clin. Nutr. 53, 1021, 1991.

[15] Ackman, R.G.; Eaton, C.A.; Sipos J.C.; Loew F.W. and Hancock, D.Comparison of fatty acid from high levels of erucic acid of RSO and partially hydrogenated fish oil in non-human primate species in a short term exploratory study. Nutr. Diet. 25; 170-185,1977. 